\title{
An exploratory study of the implementation of admission standards (child:guardian ratios) in Ontario's Class A public pools
}

\author{
Ofelia Tatar and Chun-Yip Hon \\ School of Occupational and Public Health, Ryerson University, Toronto, ON, Canada.
}

\begin{abstract}
Admission standards or specific child:guardian ratios for public pools have been endorsed and promoted by the Ontario Ministry of Health and Long Term Care (MOHLTC) to prevent recreational water injuries and fatalities. However, the voluntary adoption of these admission standards in Ontario has not been evaluated. Therefore, the aim of this study was to explore the implementation of these admission standards in Class A public pools. An online survey was developed and disseminated to Class A public pool operators in Ontario. Frequency distributions were used to describe the results. All respondents have some form of admission standards integrated into their operations, with $68 \%$ using child:parent ratios that exceed the minimum MOHLTC's recommendations. The majority of operators (87\%) felt that admission standards have a positive impact and there were no known increases in water-related incidents post-implementation. Many owners/operators (78\%) would support their enactment into the pool regulations. The findings from this study highlight the promise of utilizing admission standards to prevent or, at the least reduce, the burden of injury related to recreational water use in Ontario. While the results are encouraging, it is recommended that further research be conducted as this was an exploratory study only.
\end{abstract}

Key words: Class A public pools, admission standards, child:guardian ratios, drowning prevention, Ontario

\section{Introduction}

In Canada, drowning is the leading cause of accidental death in children 1 to 4 years of age (Canadian Institute for Health Information, 2005; Lifesaving Society, n.d.) and the second most common cause of preventable death in children under the age of 10 years (Lifesaving Society, n.d.). However, the burden of illness resulting from water safety related incidents is much greater, with up to 10 times more children experiencing near-drowning incidents that require hospitalization and one in four cases sustaining permanent brain damage (Canada Safety Council, 2009).

The number one contributing factor in fatal water incidents involving children is the lack of parental/guardian supervision of nonswimmers, predominantly those under 5 years of age (Lifesaving Society, 2015). This fact was exposed through several Coroner's inquests into the accidental drowning deaths of children (Ms. Pat Campbell, Office of the Chief Coroner, personal communication, November 2015). This revelation is consistent with research conducted by the Canadian Red Cross, which found that of all children under the age of 10 who drowned $76 \%$ were not accompanied by an adult (Canadian Red Cross, 2013).

Corresponding author: Chun-Yip Hon (email: cyhon@ryerson.ca)
To mitigate the risk of child drownings, one solution repeatedly suggested in numerous Coroner's inquests is the development and implementation of specific child to parent/ guardian ratios for swimming pools, known as Admission Standards in Ontario (Ms. Pat Campbell, Office of the Chief Coroner, personal communication, November 2015). This is supported by research identifying that adult supervision of children is instrumental in drowning prevention (Jan, 2013; Wallis et al., 2015).

Such admission standards are to be used as complementary aids to lifeguard supervision. This is because lifeguarding is highly demanding as lifeguards need to be constantly on alert and their attention fully dedicated to swimmer safety (Schwebel et al., 2010). Activities that detract a lifeguard from their normal duties (e.g., being distracted by unrelated tasks, allowing access of unsupervised small children, tolerating dangerous patron behaviour, etc.) can impede rescue efforts (Modell, 2010).

Based on the Office of the Chief Coroner's suggestion of having minimum supervisory ratios for young bathers, the Ontario Ministry of Health and Long Term Care (MOHLTC), in consultation with stakeholders, produced a set of admission standards (available at: http://www.healthunit.org/injury/swimming/ Admission_Standards_Fact_Sheet.pdf) that state the child: guardian ratios as follows: for children under the age of 6 years, 
two children to one guardian (2:1); for children aged 6-9 years, four children to one guardian (4:1) or eight children in life jackets to one guardian (8:1); and children aged 6-9 years who pass a facility swim test administered by a lifeguard may be unaccompanied.

At the MOHLTC's request, Ontario's provincial Boards of Health, through their public health units, promoted the use of these admission standards to all inspected public pool operators in hopes of reducing the risk of water-related incidents (MOHLTC, 2011).

At the time of writing, admission standards are not a legislated requirement (MOHLTC, 2012), and to our knowledge, their voluntary and discretionary adoption by Class A public pools operators in Ontario has not been explored. In Ontario Reg. 565 Public Pools, "Class A" pools include pools open to the public as well as pools at an institution or recreational camp. Therefore, the objective of this study was to evaluate the implementation of the MOHLTC's Admission Standards in Ontario's Class A public pools including the proportion of public pools that have adopted admission standards into their operations, the extent of implementation (minimum recommendations only or more stringent/additional requirements), as well as its impact on water-related incidents and on pool operations.

\section{Methods}

This was a cross-sectional study whereby data were collected through a questionnaire completed by Class A public pool owners/operators. The inclusion criteria were an individual at least 18 years of age and holding a managerial or supervisory position at a Class A pool affiliated with the Lifesaving SocietyOntario Branch (LSS-Ont). Owing to the lack of previous work on this particular matter, a de novo questionnaire was created specifically for this study. The survey questions were developed based on the objectives of the study and were related to the following three broad categories: (i) pool characteristics such as location (urban/rural), type (indoor/outdoor), and operational aspects (lifeguard information, bather load, weekly average number of bathers); (ii) admission standards particulars such as pool-specific child:guardian ratios, additional safety requirements as part of pool operation (e.g., sorting of children by swimming ability), staff training, enforcement of admission standards, and efficacy of admission standards adoption (i.e., water-related incidents pre- and post-implementation); and (iii) operator feedback related to challenges, successes, and perceived overall impact of admission standards.

Overall, there were 30 questions with varying response options available (e.g., multiple choice, dichotomous (yes/no), and Likert scale) to allow for quantitative analysis (Supplementary Appendix ${ }^{1}$ ). The survey was pretested by individuals with a background in pool safety and modifications were made based on their feedback. Ethics approval was obtained from Ryerson University prior to survey dissemination to prospective participants.

\footnotetext{
${ }^{1}$ Supplementary data are available with the article through the journal Web site at http://pubs.ciphi.ca/doi/suppl/10.5864/d2016-019.
}

On behalf of the research team, the LSS-Ont sent a recruitment email (with a link to survey) to 277 of its Class A public pool affiliates offices situated throughout the province. The online survey, hosted on SurveyMonkey, was available for completion between 25 January and 29 February 2016. A reminder email was sent to the initial list of potential participants two weeks prior to the survey's closing date.

Respondents with significantly incomplete answers were excluded from analysis. Data analysis was performed using Microsoft Excel (Seattle, WA) and descriptive statistics were used to report the results.

\section{Results}

\section{Characteristics of respondents and their pools}

Of the 277 emails sent, 139 responses were received and, of these, 25 were excluded because they were deemed significantly incomplete (i.e., answered less than $15 \%$ of the entire questionnaire). This resulted in 114 surveys included in the final analysis (response rate of $114 / 227$ or $41.2 \%$ ).

The 114 responses accounted for 145 pools, as some respondents indicated that more than one pool falls under their purview. In such instances, the responses were treated as separate observations, i.e., if a respondent indicated that they are responsible for three pools, they had three separate sets of answers.

A majority of respondents indicated that they hold a supervisory position and more than half of all respondents (58\%) have worked in their position for at least five years.

The 145 pools were relatively evenly situated between large, medium, and small/rural population centres; two-thirds were indoor pools. Nearly $90 \%$ of these pools have more certified lifeguarding staff on duty than required by legislation (i.e., R.R.O. 1990, Reg. 565: Public Pools). The majority of the pools have a maximum bather load of 100-300 and they had an average of up to 2000 bathers per week (Table 1).

\section{Admission standards particulars}

All respondents stated that they have some form of admission standards as part of their normal pool operations, and they also indicated that training on admissions standards is provided to their staff. For two-thirds of the pools, the admission standards were implemented in 2010 or earlier. Although the MOHLTC's suggestion for the age of the guardian is a minimum of 12 years of age, $60 \%$ of the respondents indicated that they require the guardian to be at least 16 years old. Twenty-two percent of respondents indicated that their pools met the Ministry's recommendations for child:guardian ratios, whereas $68 \%$ exceeded these standards, i.e., more stringent.

In addition to implementing child:guardian ratios, pool operators/owners employed other safety measures to minimize the risk of water-related incidents. The most common of these were having signage posted at the entrance of the pool and on the company's website as well as verbally explaining the child:guardian ratios to applicable patrons (Figure 1). 
Table 1. Characteristics of respondents and pool

\begin{tabular}{|c|c|}
\hline Characteristics & $\begin{array}{l}\text { No. of } \\
\text { responses* }\end{array}$ \\
\hline \multicolumn{2}{|l|}{ Job Title } \\
\hline Manager & 60 \\
\hline Supervisor & 86 \\
\hline Owner & 5 \\
\hline Operator & 16 \\
\hline $\begin{array}{l}\text { Other e.g., program coordinator, aquatics } \\
\text { coordinator, recreation director }\end{array}$ & 11 \\
\hline \multicolumn{2}{|l|}{ Years on the job } \\
\hline$\leq 1$ year & 10 \\
\hline $1-2$ years & 17 \\
\hline $2-3$ years & 8 \\
\hline $3-4$ years & 17 \\
\hline $4-5$ years & 8 \\
\hline$>5$ years & 84 \\
\hline
\end{tabular}

Pool setting ${ }^{\dagger}$

\begin{tabular}{|l|c|}
\hline Large population centre & 52 \\
\hline Medium population centre & 45 \\
\hline Small population centre & 41 \\
\hline Rural & 7 \\
\hline
\end{tabular}

Type of pool

\begin{tabular}{|l|c|}
\hline Indoor & 95 \\
\hline Outdoor & 12 \\
\hline Both indoor and outdoor & 38 \\
\hline
\end{tabular}

Maximum bather load

\begin{tabular}{|l|c|}
\hline $1-100$ & 20 \\
\hline $101-200$ & 73 \\
\hline $201-300$ & 36 \\
\hline $301-400$ & 7 \\
\hline$>400$ & 5 \\
\hline
\end{tabular}

Weekly average number of bathers

\begin{tabular}{|l|l|}
\hline $1-1,000$ & 64 \\
\hline $1,001-2,000$ & 42 \\
\hline $2,001-3,000$ & 13 \\
\hline
\end{tabular}

Table 1 (continued).

\begin{tabular}{|l|c|}
\hline Characteristics & $\begin{array}{l}\text { No. of } \\
\text { responses* }\end{array}$ \\
\hline $3,001-4,000$ & 5 \\
\hline$>4,000$ & 9 \\
\hline Number of lifeguards & \multicolumn{2}{|l}{} \\
\hline On par with legislated requirements & 14 \\
\hline Exceeds legislated requirements & 131 \\
\hline
\end{tabular}

"A respondent could answer in more than one sub-category ( $n=114$ respondents).

${ }^{\dagger}$ As defined by Statistics Canada: large $\geq 100,000$ people; medium =30,000-99,999; small $=1,000-29,999 ;$ and rural $<1,000$.

${ }^{\star}$ As per RRO 1990 Reg. 565 Public Pools.

When supervision ratios are not met by incoming pool patrons, the majority of pools (86\%) do not grant admission. However, a small proportion of pools have no policy on this matter $(6 \%)$ and, in some cases (1\%), the owner/operator allows patrons into the pool but only at their own risk.

With regards to the impact of admission standards on waterrelated incidents, there were no known increases. However, as shown in Figure 2, the reported outcomes with respect to water-related incidents are mixed.

\section{Operator feedback}

The majority of operators (87\%) described the overall impact of admission standards on their operations as being "positive" or "very positive." Respondents indicated that the key benefits associated with implementation of admission standards were improved water safety and that they facilitated the supervision of younger swimmers. Nevertheless, pool owners/operators did report challenges with respect to implementation of admission standards with the two most common reported concerns being (i) increased processing time of patrons and (ii) pushback or complaints from the public (Figure 3 ).

Despite these challenges, more than three-quarters (78\%) of respondents were in support of making admission standards mandatory.

\section{Discussion}

This was an exploratory study examining the state of admission standards or specific adult supervision criteria in Class A public pools in Ontario used to improve water safety. All respondents claimed to have admission standards integrated in their pool operations. Approximately two-thirds of the respondents had child:guardian ratios $(68 \%)$ as well as a minimum guardian age that exceeded the MOHLTC's recommendations (60\%). Perhaps this reflects the perceptions of pool owners/operators that the Ministry's recommendations are insufficient. Alternatively, it could mean that pool owners/operators have a heightened understanding of the crucial role that adult supervision plays in the prevention of recreational water incidents in children. This is 


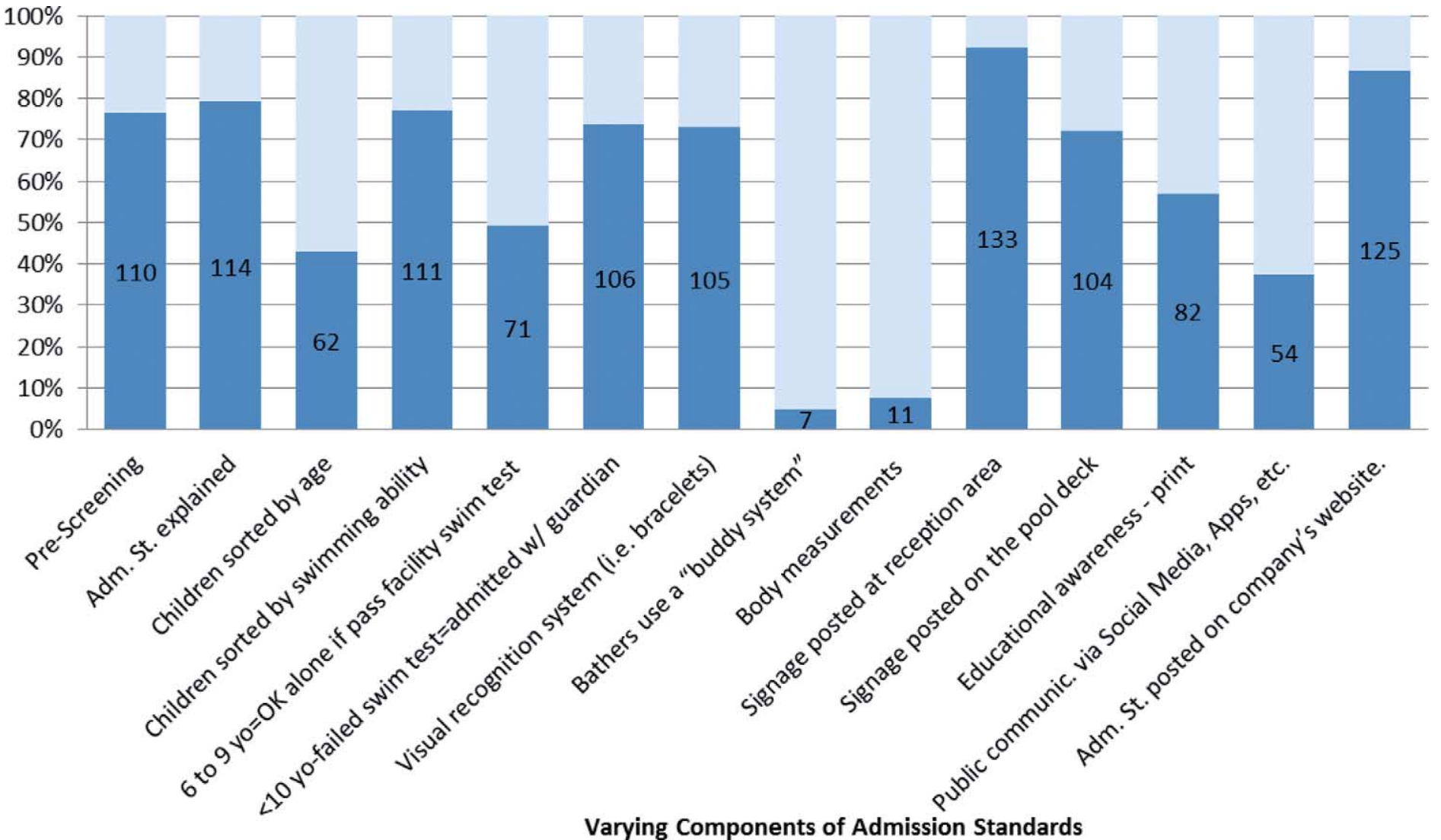

Figure 1. Elements of pool admission standards other than child:guardian ratios employed by respondents.

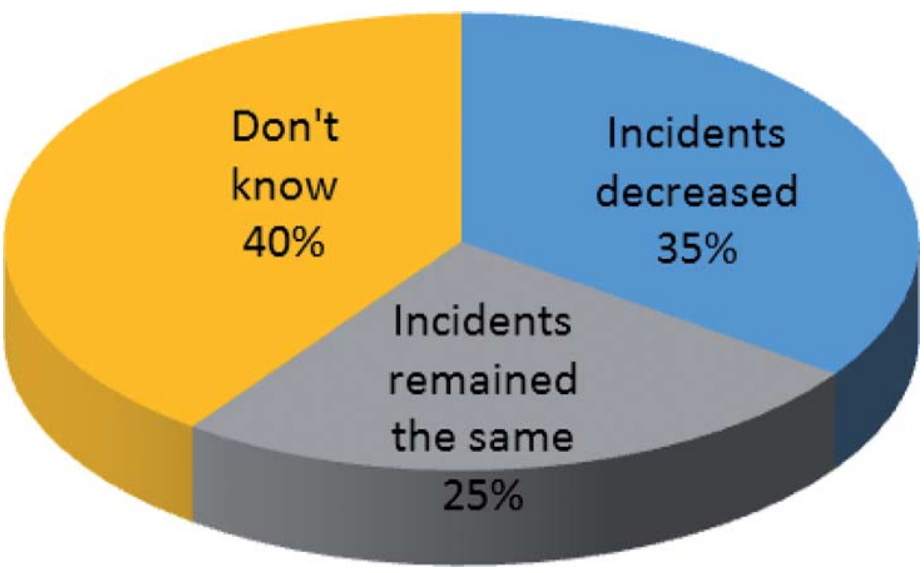

Figure 2. Reported impact on the number of waterrelated incidents (e.g., slips, falls, near-drowning or drowning occurrences, etc.) after the implementation of admission standards.

supported by the fact that a large majority of respondents (90\%) have more certified lifeguarding staff on duty than required by law. Voluntarily surpassing the minimum requirements likely infers that maintaining water safety, especially for children, is of great importance to owner/operators.

No pools reported an increase in the overall number of water-related safety incidents pre- and post-admission standards implementation. This is an important finding; however, whether there were actual decreases in water-related incidents is unclear

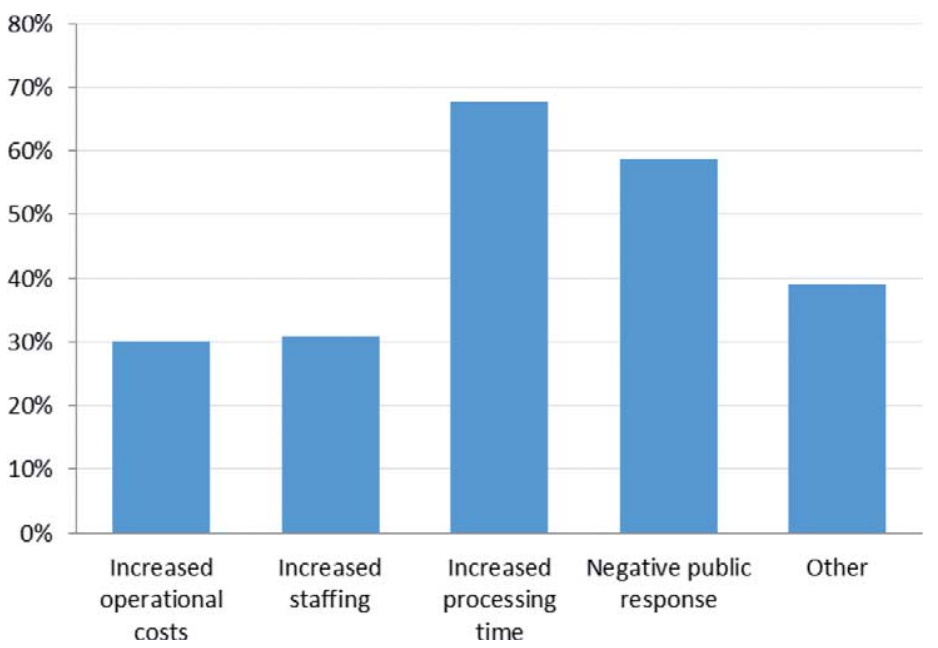

Figure 3. Reported challenges by operators with respect to implementation of admission standards. "Other" responses include: lack of standardization across pools that leads to customers' complaints, difficulties in educating staff in dealing with disgruntled patrons, and perceived discrimination experienced by families with many small children that do not meet the child:guardian ratios.

and a long-term study of time trends is recommended to confirm this.

With respect to operator feedback regarding admission standards, the majority of respondents indicated improved safety and support of lifeguarding duties as the top successes, 
this suggests that these admission standards are meeting their intended purpose. There were, however, some unfavourable aspects with admission standards implementation with more than $50 \%$ of respondents reporting increased processing time of patrons and public disagreement as the main challenges. Nevertheless, it appears that the challenges are manageable and that the successes associated with admission standards (e.g., improved safety and assists lifeguards in performing their duties) outweigh the negatives. This is reflected as a majority of respondents $(87 \%)$ described the overall impact of admission standards on their operations as positive or very positive.

In addition to child:guardian ratios, we discovered that other elements of pool admission were used by more than $50 \%$ of the respondents to improve water safety. This includes prescreening at entry (e.g., age of child and (or) guardian), sorting of children by swimming ability (e.g., swim test), use of a visual recognition system (e.g., coloured bracelets), and posting of signage on premises (e.g., on pool deck, at the entrance and (or) change room). This assortment of safeguards that are employed in varying combinations sheds light on the lack of standardization across Ontario, which was identified by respondents as a contentious point in their relationship with the public. This might explain why owners/operators strongly support having admission standards made legally binding.

With regards to water safety legislation, the landscape is remarkably diverse and inconsistent mainly due to the variety of approaches by health authorities across multiple jurisdictions (Crawford et al., 2014; Liguori et al., 2014). Specifically in Canada, our review found that of the 13 provinces and territories only Alberta had pool admission standards incorporated in its legislation (Alberta Government, 2014). This is noteworthy as the literature suggests that drowning death rates decreased following implementation of relevant water safety acts and regulations (Leavy et al., 2016; Pearn et al., 2008; Szpilman et al., 2012). Although safety legislation is credited with a reduction in accidental drowning deaths in children, it is recognized that for sustained, long-term effectiveness additional strategies need to be utilized such as on-going advocacy, public awareness campaigns, and enforcement of legislative requirements (Leavy et al, 2016; Pearn et al., 2008).

Some study limitations need to be addressed. Selection bias is a potential issue as we recruited only LSS-OB affiliates to participate in the study. It is possible that since LSS supports and promotes admissions standards their affiliates might be more likely to implement admission standards compared with nonaffiliates. This concern was alleviated by the fact that a significant number of Class A public pools in Ontario are affiliated with LSS.

There is also possible recall bias on the part of respondents, specifically the question about when admission standards were adopted and the number of water-related incidents postimplementation. Given the concern with the latter, we recommend a future study examining the time trends of incidents. Another bias is that the respondents were primarily pool owners/operators. It would be important to gain the perspective of other pool stakeholders such as lifeguards and possibly members of the public, prior to making a decision about legislating admission standards. A final bias is that this study only examined Class A public pools and excluded Class B pools. As the latter are unsupervised, the current findings may not be reflective of Class B pool operators/owners. This was an inevitable consequence of time and resource limitations. It is recommended that future similar studies examine admission standards implementation at all types of pools in Ontario.

Another study limitation is that the survey did not account for the possibility of one operator providing responses for multiple pools as it was unbeknownst to the research team at the time of questionnaire development that a potential respondent may be responsible for more than one pool. In future studies that employ this same questionnaire, it is recommended that there be an additional question that would allow respondents to select the number of pools they represent at the time of the survey. Lastly, as the data were self-reported, we are not able to verify the responses.

In conclusion, the results of this exploratory study found that even though admission standards are currently voluntary in Ontario, all pool owners/operators that responded to the survey have adopted some form of admission standards in their operations. In fact, most have exceeded the MOHLTCs recommendations. That so many pool operators surpass the minimum recommended criteria highlights an elevated awareness with regards to the importance of adult supervision of children. Given these findings, we are confident in stating that owners/operators would be amenable if admission standards were to be prescribed in legislation.

The findings from this study could be used by public pool operators, prevention leaders (i.e., LSS), and policy makers, with the goal of reducing/preventing the burden of injury and illness related to recreational water use. However, it bears mentioning that in addition to admission standards a variety of antidrowning initiatives, including structural and educational interventions, should be employed to ensure effectiveness of the prevention strategy (Crawford et al., 2014; Leavy et al., 2016). Furthermore, when developing these strategies in Ontario, the reality and particulars of an increasingly diverse population need to be considered, and to be effective, the intervention and its execution ought to be culturally mindful (Gallinger et al., 2015). Although the overall uptake of admission standards in Ontario is positive and they appear to be effective, additional research should be conducted for conclusive results.

\section{Acknowledgements}

The authors wish to thank Michael Shane and Nathalie Vallières of the Royal Lifesaving Society Canada, Ontario Branch, who assisted with the dissemination of the survey. We are also grateful to all the Class A public pool operators who completed the survey.

\section{References}

Alberta Government. 2014. Pool standards. Available at: http:// www.health.alberta.ca/documents/Standards-Pools.pdf [accessed 12 October 2015]. 
Canada Safety Council. 2009. Keep your head above water. Available at: https://canadasafetycouncil.org/campaigns/keep-your-head-abovewater [accessed 12 October 2015].

Canadian Institute for Health Information. 2005. Helping Canadians stay healthy and safe. CIHI Direction ICIS. 12(3). Available at: http://publications.gc.ca/collections/collection_2007/cihi-icis/H1161-12-3E.pdf [accessed 12 October 2015].

Canadian Red Cross. 2013. Facts \& figures, water safety week 2013. Available at: http://www.redcross.ca/crc/documents/What-We-Do/ Swimming-Water-Safety/facts-and-figures-water-safety-week-2013. pdf [accessed 12 October 2015].

Crawford, G., Leavy, J., Portsmouth, L., Jancey, J., Leaversuch, F., Nimmo, L., et al. 2014. Development of a systematic review of public health interventions to prevent children drowning. Open J. Prev. Med. 04(03): 100-106. doi: 10.4236/ojpm.2014.43014

Gallinger, Z.R., Fralick, M. and Hwang, S.W. 2015. Ethnic differences in drowning rates in Ontario, Canada. J. Immigrant Minority Health. 17(5): 1436-1443. doi: 10.1007/s10903-014-0095-7

Jan, M.M. 2013. Pediatric near-drowning and drowning. Saudi Med. J. 34(2): 119-122.

Leavy, J., Crawford, G., Leaversuch, F., Nimmo, L., McCausland, K. and Jancey, J. 2016. A review of drowning prevention interventions for children and young people in high, low and middle income countries. J Commun. Health. 41(2): 424-441.

Lifesaving Society. 2015. Ontario drowning report 2015. Available at: http://www.lifesavingsociety.com/media/216840/98drowning report2015ontario_web.pdf [accessed 12 October 2015].

Lifesaving Society. n.d. Who's drowning. Available at: http:// www.lifesavingsociety.com/who\%E2\%80\%99s-drowning.aspx [accessed 12 October 2015].
Liguori, G., Capelli, G., Carraro, E., Di Rosa, E., Fabiani, L., Leoni, E., et al. 2014. A new checklist for swimming pools evaluation: A pilot study. Microchem. J. 112: 181-185. doi: 10.1016/j.microc.2013. 09.018

Ministry of Health and Long Term Care (MOHLTC). 2011. Letter to all owners/operators and lifeguards of public pools. Available at: http://www.ciphi.on.ca/images/stories/pdf/advocacy/moh_ admission_standards.pdf [accessed 13 October 2015].

Ministry of Health and Long Term Care (MOHLTC). 2012. Admission standards fact sheet. Available at: http://www.health unit.org/injury/swimming/Admission_Standards_Fact_Sheet.pdf [accessed 13 October 2015].

Modell, J.H. 2010. Prevention of needless deaths from drowning. Southern Med. J. 103(7): 650-653. doi: 10.1097/SMJ.0b013 e3181e10564

Pearn, J.H., Nixon, J.W., Franklin, R.C. and Wallis, B. 2008. Safety legislation, public health policy and drowning prevention. Int. J. Injury Control Safety Promo. 15(2): 122-123. doi: 10.1080/ 17457300802150587

Schwebel, D.C., Jones, H.N., Holder, E. and Marciani, F. 2010. Lifeguards: A forgotten aspect of drowning prevention. J Injury Violence Res. 2(1): 1. doi: 10.5249/jivr.v2i1.32

Szpilman, D., Bierens, J., Handley, A.J. and Orlowski, J.P. 2012. Current concepts: Drowning. N. Engl. J. Med. 366(22): 21022110. doi: $10.1056 /$ NEJMra1013317

Wallis, B.A., Watt, K., Franklin, R.C., Taylor, M., Nixon, J.W. and Kimble, R.M. 2015. Interventions associated with drowning prevention in children and adolescents: Systematic literature review. Injury Prev. 21(3): 195-204. doi: 10.1136/injuryprev2014-041216 